\title{
Stochastic Finite Element Analysis of the Free Vibration of Laminated Composite Plates
}

\author{
Shaker, A., \\ Department of Aerospace Engineering, Cairo University, Cairo, Egypt. \\ Sadek, E., \\ Department of Aerospace Engineering, Cairo University, Cairo, Egypt. \\ Tawfik, M., \\ Department of Mathematical Sciences, German University in Cairo, Cairo, Egypt. \\ and Abdelrahman, W. G., \\ Department of Aerospace Engineering, King Fahd University of Petroleum and Minerals, \\ Dhahran, Saudi Arabia
}

\begin{abstract}
Using the Stochastic Finite Element Method (SFEM) to perform reliability analysis of the free vibration of composite plates with material and fabrication uncertainties has received much attention lately. In this work the stochastic analysis is performed using the First Order Reliability Method (FORM-method 2) and the Second Order Reliability Method (SORM). The basic random variables include laminae stiffness properties and material density, as well as the randomness in ply orientation angles. Modeling of the composite behavior utilizes a nine-noded isoparametric Lagrangian element based on the third order shear deformation theory. The developed code utilizes MATLAB capabilities to derive the derivatives of the reduced stiffness and mass matrices symbolically with a considerable reduction in calculation time. Calculating the eigenvectors at the mean values of the variables proves to be a reasonable simplification which significantly increases solution speed. The stochastic finite element code is validated using available data in the literature, in addition to comparisons with results of the well-established Monte Carlo simulation technique with importance sampling. Results show that SORM is an excellent rapid tool in the stochastic analysis of free vibration of composite plates, when compared to the slower Monte Carlo simulation techniques.
\end{abstract}

\section{Introduction}

Dynamic behavior of composite laminates is a function of the geometrical and material properties of these laminates. Mechanical properties, density, stacking sequence, as well as the laminate dimensions determine the values of the natural frequencies. These quantities are not deterministic in nature. Rather, uncertainties in their values due to manufacturing and fabrication result in variations in the behavior characteristics of the laminate such as the values of the natural frequencies. Computer simulations of composite laminates used in aerospace applications often show closely packed or overlapping natural frequencies. In such cases even the slightest shift in characteristics of the laminate can have a pronounced effect on the response of the structure. For proper 
quality control of the dynamic characteristics of laminates, their sensitivities to the laminate properties need to be investigated.

Analysis of structures with deterministic characteristics to random excitations has been reported extensively in the literature; see [1] for example. This is not the case, however, for the analysis of composite structures with a comprehensive implementation of uncertainties. Sources of uncertainties range from the statistical nature of the material properties of the constituents, to the inevitable fabrication randomness in layup and curing. To implement the effects of material and manufacturing uncertainties, a set of random variables is chosen and the randomness in these variables is quantified experimentally or using simulation codes. These random variables are usually chosen as the composite laminate mechanical properties, density, and orientation angles. Ibrahim [2] and Manohar and Ibrahim [3] has presented a review of structural dynamics problems with such stochastic parameter variations. Oh and Librescu [4] developed a meancentered second-moment method to study the free vibration and reliability of composite cantilevers.

Composite plates were also analyzed using these techniques. In their work, Salim et al. [5-7] have employed a First Order Perturbation Technique FOPT to perform static analysis of composite plates using classical laminated plate theory with random material properties. The static response of uncertain FEM discretised structures was evaluated by Falsone and Impollonia [8], while Chamis et al. [9,10] combined optimization techniques with probabilistic structural analysis to reduce the maximum deflection of a composite simulated fuselage by a factor of 4.5 .

Several higher order finite elements have been employed to model plate dynamic response in most of such analyses. Elseifi [11] used a four noded element based on a higher order shear deformation theory to perform reliability analysis of thick composite plates subject to first-ply failure with parameter uncertainties. Shankara and Iyengar [12] used a higher order shear deformation theory to formulate elements with 5 DOFs and 7 DOFs per node to study the free vibration of plates. Later, Singh et al. [13] added the complexity of random material properties to the free vibration analysis of composite plates.

The aim of the present work is to study and develop a Stochastic FEM code to investigate the fundamental frequency of rectangular composite plates made up of laminae with uncertain parameters. The generalized analysis allows the random variables representing material properties to be normal or nonnormal, correlated or uncorrelated. Laminate mechanical behavior is modeled using a higher order shear deformable element. Considerable reduction in calculation time is achieved by deriving the derivatives of the reduced stiffness and mass matrices symbolically. The code is built using the MATLAB 7.1 compiler and all runs are made on a P4 $2.8 \mathrm{GHz}$ machine with 512 MB RAM.

\section{Reliability Models of the Composite Laminate}

The commonly used deterministic safety factors do not provide adequate information to achieve optimal use or resources, while probabilistic analysis does. The first step in studying the variation of the fundamental frequency of the composite plate,

$\omega_{p}$, due to uncertainties in its material properties and ply angles is to define a suitable and 
specific performance criterion. The plate $a \times b \times h$ composite laminate, shown in Fig. 1, is assumed to be subjected to a periodic load with frequency $\omega_{L}$, which can take any value up to $\omega_{p}$. In probabilistic design, $\omega_{p}$ is not a unique value, but has a certain distribution. This distribution can be quantified by its mean value and standard deviation. At the design point, the plate fundamental frequency $\omega_{p}$, is equal to a certain specified value $\omega_{r}$, which can be taken as that of the periodic load. Accordingly, a suitable performance function is defined as:

$$
g(X)=\left(\lambda_{p} / \lambda_{r}\right)-1
$$

where $\lambda_{p}, \omega_{r}=\omega_{p}^{2}$, are the eigenvalues, and $X$ is a vector of basic random variables, given by:

$$
X=\left[E_{11}, E_{22}, G_{31}, G_{23}, G_{12}, v_{12}, \rho, \theta_{1}, \theta_{2}, \ldots, \theta_{m}\right]
$$

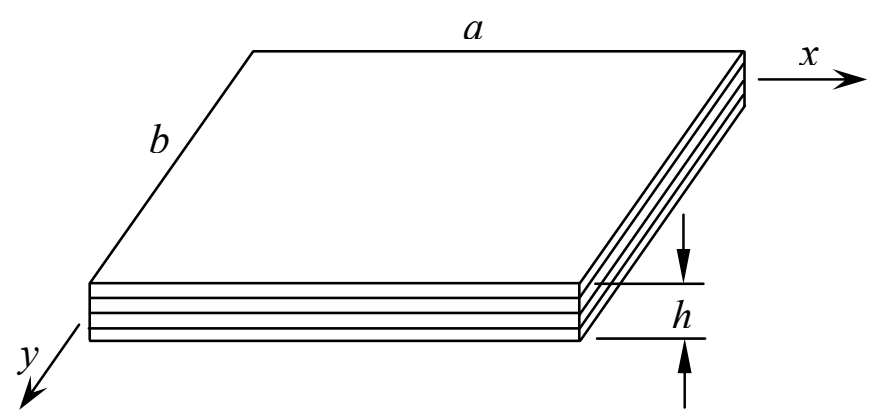

Fig. 1. Geometry of the composite plate.

Here, $E_{11}, E_{22}, G_{12}, G_{13}, G_{23}$ and $v_{12}$ are the mechanical properties of each lamina in its principal directions, $\rho$ is the material density, and $\theta_{i}$ are the ply orientation angles. A failure surface or a limit state of interest can then be defined as $g(X)=0$, with the probability of failure calculated from:

$$
p_{f}=\int \ldots \int_{g<0} f_{X}\left(X_{1}, X_{2}, \ldots, X_{n}\right) d X_{1} d X_{2} \ldots d X_{n}
$$

where $f_{X}\left(X_{1}, X_{2}, \ldots, X_{n}\right)$, is the joint probability density function for the $n$ basic random variables, where $n=7+m$. The integration is performed over the failure region $g()<0$.

There are several methods to calculate $p_{f}$. Here we shall use two types of analytical approximations that lead to two methods; the First-Order Reliability Method (FORMMethod 2), and the Second-Order Reliability Method (SORM). Both methods will be investigated in their ability to correctly predict the probability of failure and the Most Probable Point (MPP) of the system. A detailed account of both methods can be found in [17] and will be summarized here. 


\section{First-Order Reliability Method (FORM Method-2):}

In this method a Newton-type recursive formula is used to find the design point when the performance unction is implicit, as in the case when using finite element formulation to describe the behavior of the system. First the vector $X$ is transformed into a reduced $X^{\prime}$ with normal random variables of zero mean and unit standard deviation. The starting point of the procedure in the space of $X^{\prime}$ is usually taken as the point of mean values. This point does not, in general, lie on the limit surface $g\left(X_{0}^{\prime}\right)=0$, as shown in Fig. 2 for a two dimensional space. The equation of the limit state is now linearized around $X_{0}^{\prime}$ :

$$
g(X)=c_{0}+\nabla g^{T}\left(X_{0}^{\prime}\right) X^{\prime}
$$

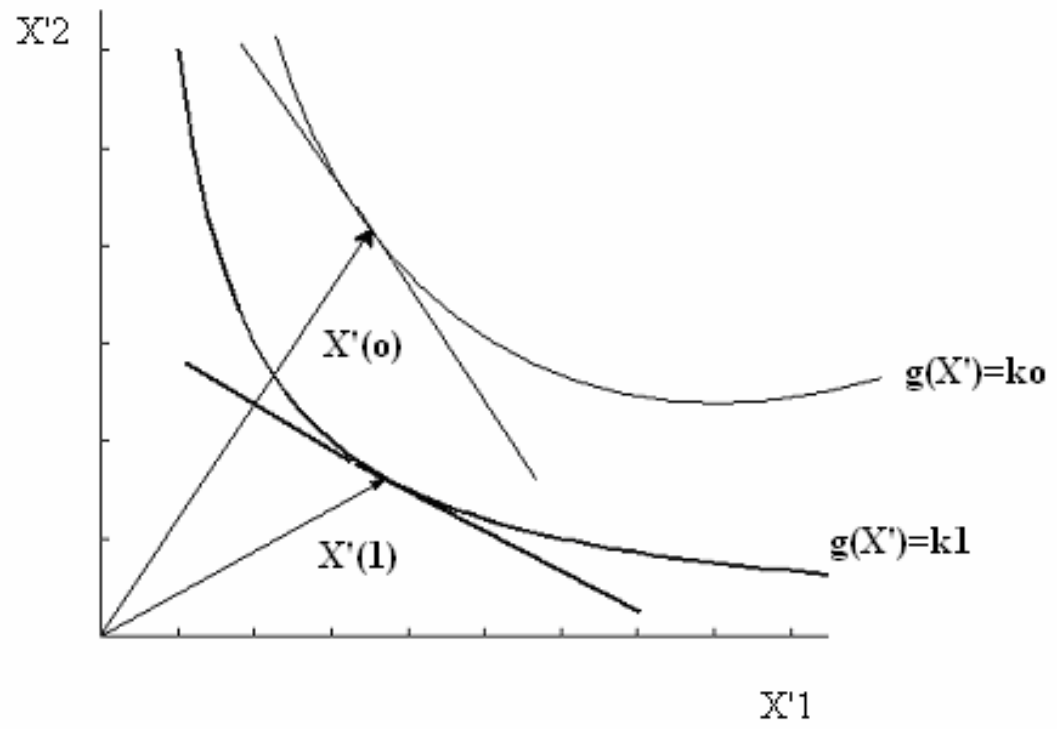

Fig. 2. FORM Method-2 for the nonlinear limit state.

Since the performance function is nonlinear, then its gradient is not constant. In this case, the new design point is obtained recursively by:

$$
X_{k+1}^{\prime *}=\frac{1}{\left|\nabla g\left(X_{k}^{\prime *}\right)\right|^{2}}\left[\nabla g^{T}\left(X_{k}^{\prime^{*}}\right) X_{k}^{\prime *}-g\left(X_{k}^{\prime *}\right)\right] \nabla g\left(X_{k}^{\prime *}\right)
$$

The distance from the origin to this new design point in the $X^{\prime}$-space is:

$$
\beta=\sqrt{\sum_{i=1}^{n} X_{i}^{\prime * 2}}
$$

The procedure is terminated when reaching the Most Probable Point (MPP). MPP is 
assumed to be reached when both of the following conditions are satisfied

$$
\begin{aligned}
& \left|\beta_{k+1}-\beta_{k}\right| \leq \varepsilon, \\
& \left|g\left(X_{k+1}^{\prime *}\right)\right| \leq \delta,
\end{aligned}
$$

with $\varepsilon$ and $\delta$ being reasonably small numbers. The probability of failure in this case is:

$$
p_{f}=1-\Phi(\beta)
$$

where $\Phi$ is the cumulative distribution function of a standard normal distribution with zero mean and unit standard deviation.

\section{Second-Order Reliability Method (SORM):}

This method uses a second order Taylor approximation of the nonlinear limit state function in order to better model its curvature. This expansion at a given point $X^{*}$ in the standard normal variable space is:

$$
g\left(X_{1}\right) \cong g\left(X^{*}\right)+\sum_{i=1}^{n} \frac{\partial g}{\partial X_{i}}\left(X_{i}-X_{i}^{*}\right)+\frac{1}{2} \sum_{i=1}^{n} \sum_{j=1}^{n}\left(X_{i}-X_{i}^{*}\right)\left(X_{j}-X_{j}^{*}\right) \frac{\partial^{2} g}{\partial X_{i} \partial X_{j}}(9)
$$

A simple closed-form solution for the probability of failure using this second-order approximation is derived using the theory of asymptotic approximations in [18] as:

$$
p_{f}=\Phi(-\beta) \prod_{i=1}^{n-1}\left(1+\beta \kappa_{i}\right)^{-0.5}
$$

where $\beta$ is the reliability index using FORM, and $\kappa_{i}$ are the principal curvatures of the limit state at the minimum distance point. These curvatures are obtained as follows. First the $X^{\prime}$ standard normal variables are rotated to another set of coordinates, denoted as $Y$, such that the last component of the new set, $Y_{n}$, coincides with $\alpha$, the unit gradient vector of the limit state at the design point. This transformation is shown in Fig. 3 for the case of $n=2$. This orthogonal transformation is given by:

$$
Y=R X^{\prime}
$$

where $R$ is the rotation matrix. For the case $\mathrm{n}>2$, the rows $r_{i}$ of this matrix are calculated using Gram-Schmidt orthogonalization procedure, see [17], as:

$$
\begin{aligned}
& r_{n}=r_{0 n}, \\
& r_{k}=r_{0 k}-\sum_{j=k+1}^{n}\left[\frac{r_{j} r_{0 k}^{T}}{r_{j} r_{j}^{T}} r_{j}\right], \quad k=n-1, n-2, . ., 1
\end{aligned}
$$


where $r_{0 i}$ are the rows of the matrix $R_{0}$, given by:

$$
R_{0}=\left[\begin{array}{cccc}
1 & 0 & \cdots & 0 \\
0 & 1 & \cdots & 0 \\
0 & \cdots & \cdots & \cdots \\
\alpha_{1} & \alpha_{2} & \cdots & \alpha_{n}
\end{array}\right],
$$

with $\alpha_{l}$ being the components of the unit gradient vector $\alpha$ at the design point.

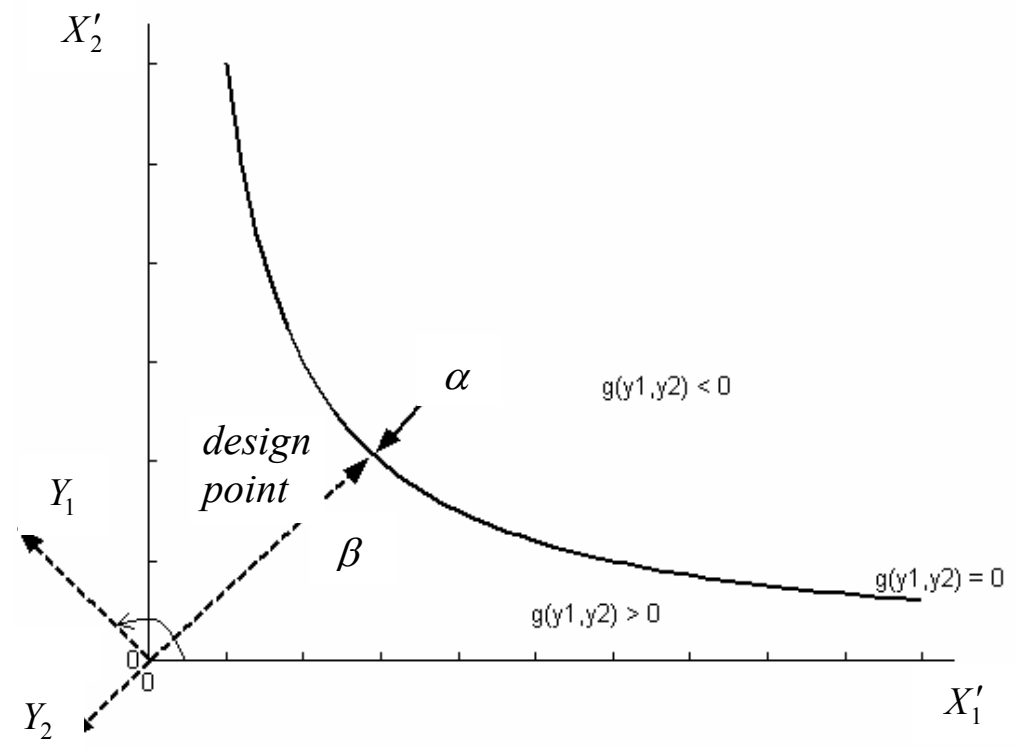

Fig. 3. SORM rotation of coordinates.

Defining a matrix A whose elements are denoted by $a_{i j}$ is computed as:

$$
a_{i j}=\frac{\left(R D R^{T}\right)_{i j}}{\left|\nabla g\left(Y^{*}\right)\right|}
$$

where $D$ the $n \times n$ second-derivative matrix of the limit state surface in the standard normal space evaluated at the design point. Since $Y_{n}$ coincides with the $\beta$-vector computed in FORM, the last column and last rows in the $A$ matrix and the last row in the $Y$ vector are dropped out to take this factor into account. The limit state can then be rewritten in terms of a second-order approximation in the rotated $Y$ space as:

$$
Y_{n}=\beta+\frac{1}{2} Y^{T} A Y,
$$

where $A$ is now of size $(n-1) \mathrm{x}(n-1)$. The required curvatures $\kappa_{i}$ are computed as the eigenvalues of the matrix $A$. The probability of failure can now be calculated from Eq. (10). 


\section{Finite Element Model}

To include transverse shear stresses and rotatory inertia effects, several shear deformation theories are developed. Here, an element based on the Higher-Order Shear Deformation Theory (HSDT) is utilized. Development of the element, detailed in [13] and briefly summarized here, employs the parabolic shear deformation theory.

\section{Displacement Field}

In the parabolic shear deformation theory, the displacement field is described in terms of midsurface displacements $u, v$ and $w$, the perpendicular to the midplane, $\zeta$, and the rotations of the normal to the midsurface at $\zeta=0, \phi_{1}$ and $\phi_{2}$. Considering the derivatives of the out-of-plane displacement as separate independent degrees of freedom transforms this system, with 5 degrees of freedom per node and $C^{1}$ continuity, into one with 7 degrees of freedom per node and mathematically easier $C^{0}$ continuity. The displacement field may be modified to accommodate $C^{0}$ continuity, see [12]. The resulting displacement field is:

$$
\begin{aligned}
& \bar{u}\left(x_{1}, x_{2}, \zeta, t\right)=u+f_{1}(\zeta) \phi_{1}+f_{2}(\zeta) \theta_{1} \\
& \bar{v}\left(x_{1}, x_{2}, \zeta, t\right)=v+f_{1}(\zeta) \phi_{2}+f_{2}(\zeta) \theta_{2} \\
& \bar{w}\left(x_{1}, x_{2}, \zeta, t\right)=w
\end{aligned}
$$

where:

$\theta_{1}=\partial w / \partial x_{1}, \theta_{2}=\partial w / \partial x_{2}, f_{1}(\zeta)=\zeta-4 \zeta^{3} / 3 h^{2}$, and $f_{2}(\zeta)=-4 \zeta^{3} / 3 h^{2}$

which satisfies the conditions of stress-free upper and lower plate surfaces.

\section{$\underline{\text { Strain Energy }}$}

The elastic strain energy of the laminated composite plate as it undergoes deformation is:

$$
U=\frac{1}{2} \int_{A} \bar{\varepsilon}^{T} D \bar{\varepsilon} d A
$$

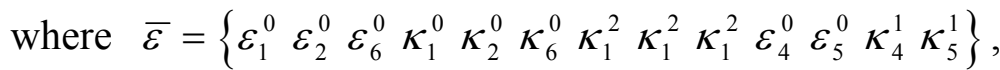

$$
D=\left[\begin{array}{ccccc}
A 1 & B 1 & E & 0 & 0 \\
B 1 & D 1 & F 1 & 0 & 0 \\
E & F 1 & H & 0 & 0 \\
0 & 0 & 0 & A 2 & D 2 \\
0 & 0 & 0 & D 2 & F 2
\end{array}\right],
$$

with

$$
\begin{aligned}
& \left(A 1_{\mathrm{ij}}, B 1_{\mathrm{ij}}, D 1_{\mathrm{ij}}, E_{\mathrm{ij}}, F 1_{\mathrm{ij}}, H_{\mathrm{ij}}\right)=\sum_{k=1}^{n} \int_{\zeta_{k-1}}^{\zeta_{k}} Q_{\mathrm{ij}}^{(k)}\left(1, \zeta, \zeta^{2}, \zeta^{3}, \zeta^{4}, \zeta^{6}\right) d \zeta, \\
& \left(A 2_{\mathrm{ij}}, D 2_{\mathrm{ij}}, F 2_{\mathrm{ij}}\right)=\sum_{k=1}^{n} \int_{\zeta_{k-1}}^{\zeta_{k}} Q_{\mathrm{ij}}^{(k)}\left(1, \zeta^{2}, \zeta^{4}\right) d \zeta,
\end{aligned}
$$


where $i$ and $j$ take the values 1,2,6 in (21.a) and take the values 4,5 in (21.b). In these equations, the orthotropic lamina stiffnesses are given by:

$$
Q_{11}=\frac{E_{11}}{d}, Q_{22}=\frac{E_{22}}{d}, Q_{12}=\frac{v_{12} E_{22}}{d}, Q_{44}=G_{23}, Q_{55}=G_{13}, Q_{66}=G_{12}
$$

where $d=1-v_{12} v_{21}$.

The strain energy functional is computed for each element and then summed over all the elements in the domain to get the total functional for the domain. Following this procedure, Eq. (18) can be written as:

$$
U=\sum_{e=1}^{N E} U^{(e)}=\sum_{e=1}^{N E} \frac{1}{2} \int_{A^{(e)}} \bar{\varepsilon}^{T} D \bar{\varepsilon} d A
$$

where, $N E$ is the number of elements. Upon substituting for the strain vector, the mechanical strain energy becomes:

$$
U=q^{T} K q
$$

where $K$ is the global stiffness matrix and $q$ is the global displacement vector.

\section{Kinetic Enerqy}

The kinetic energy of the vibrating plate, within the domain of small displacements, is:

$$
T=\frac{1}{2} \int_{A} \sum_{k=1}^{N L} \int_{\zeta_{k-1}}^{\zeta_{k}}\left(\rho^{(k)} \dot{\hat{u}}^{T} \dot{\hat{u}}\right) d \zeta d A
$$

where $\hat{u}$ is the displacement vector given by $\hat{u}=\{\bar{u} \bar{v} \bar{w}\}$ and $\rho^{(k)}$ is the density of layer $k$. Similar to the strain energy, this expression can be rewritten as:

$$
T=\dot{q}^{T} M \dot{q}
$$

where $M$ is the global mass matrix.

\section{FEM Formulation}

Using variational principles, the governing equations for free vibration for the system can be derived as:

$$
K q+M \ddot{q}=0
$$

For positive definite $M$, Eq. (27) can be transformed into a standard eigenvalue problem: 


$$
A q-\lambda q=0,
$$

where $A=M^{-1} K$ and $\lambda_{P}=\omega_{p}{ }^{2}$, with $\omega_{p}$ being the natural frequency of the plate. This FEM formulation, augmented with suitable boundary conditions, is used next to represent the system response when calculating the implicit objective function at each iteration of the stochastic analysis.

\section{Stochastic Finite Element Analysis}

In reliability analysis, the partial derivatives of the performance function $g(X)$ with respect to all random variables $X_{i}$ are required. These can be expressed, using the chain rule and Eq. (1) as:

$$
\frac{\partial g}{\partial X_{i}}=\frac{1}{\lambda_{r}} \frac{\partial \lambda_{p}}{\partial X_{i}}, \quad i=1,2, . . n
$$

The partial derivatives of the $j$ th eigenvalue with respect to the random variables have been derived in by Hasselman and Hart [14] as:

$$
\frac{\partial \lambda_{j}}{\partial X}=\frac{\frac{\partial}{\partial X}\left[\phi_{j}^{T}\left(K-\lambda_{j} M\right) \phi_{j}\right]}{\phi_{j}^{T} M \phi_{j}},
$$

where $\phi_{j}$ is the eigenvector corresponding to $\lambda_{j}$.

Noting that $K$ is independent of $\rho$, while $\rho$ is a common factor of all elements of $M$, substitution of Eq. (30) into Eq. (29) yields:

$$
\begin{aligned}
\frac{\partial g}{\partial X_{i}} & =\frac{1}{\lambda_{r}} \frac{\frac{\partial}{\partial X_{i}}\left[\phi_{p}^{T} K \phi_{p}\right]}{\phi_{p}^{T} M \phi_{p}}, \text { for } i=1,2, . .6,8,9, . . n, \\
\frac{\partial g}{\partial X_{7}} & =\frac{-\lambda_{p}}{\lambda_{r} \rho} .
\end{aligned}
$$

Standard finite difference routines can be used to evaluate the derivatives of the stiffness matrix $K$ in Eq. (31.a) with respect to the random variables. This, however, becomes time consuming, especially when the set of random variables include ply orientation angles, because the process is repeated at each iteration point. Moreover, since the prediction of the new point depends on the derivatives, which are approximate in this case, the optimization method takes a larger number of iterations to converge. Finally, using SORM in computing the probability of failure requires calculating the second derivatives as well, which deems the finite difference choice impractical. Use is made of MATLAB symbolic capabilities in evaluating the derivatives of the reduced stiffness and mass matrices. In evaluating the derivative in the numerator of Eq. (31), the 
eigenvectors at the mean value of $X$ are used, and are not updated at each iteration. This greatly simplifies calculations and is justifiable for large frequency ratios. As the frequency ratio increases, MPP tends to be closer to the mean value of $X$, which is used in calculating the eigenvectors. Validity of this simplification, and confidence in the whole modeling, is further established with comparisons with available published results and with results obtained when this simplification is not used.

For the case when all the variables are treated as uncorrelated random variables, the eigenvalue can be assumed to have a statistical distribution with mean and variance calculated at the mean of the random variables, given by:

$$
\begin{aligned}
& \mu_{\lambda} \simeq \lambda\left(\mu_{X_{1}}, \mu_{X 2}, \ldots \mu_{X_{n}}\right) \\
& \operatorname{Var}(\lambda)=\sigma_{\lambda}^{2} \simeq \sum_{i=1}^{n}\left(\frac{\partial \lambda}{\partial X_{i}}\right)^{2} \operatorname{Var}\left(X_{i}\right)
\end{aligned}
$$

\section{Numerical illustrations}

\section{Second Order Statistics of a square laminate:}

The first illustration is a square symmetric $\left[0^{\circ} / 90^{\circ}\right]_{\mathrm{s}}$ laminate with $a / h=10$. Only natural frequencies and second order statistics are investigated for this laminate in [13] for SSSS boundary conditions. The mean values of the material properties are: $E_{11} / E_{22}=25, G_{13} / E_{22}=G_{12} / E_{22}=0.5, G_{23} / E_{22}=0.2, E_{22}=10.3 \mathrm{GPa}, v_{12}=0.25, \rho=1$.

Table 1 shows a comparison of the first five calculated nondimensional natural frequencies of the laminate using two values for the mesh size and those reported in [13]. It is clear from the table that a good agreement is obtained for the $2 \times 2$ mesh and excellent agreement is obtained using the fine $5 \times 5$ mesh, with a difference of only $1.35 \%$ in the fifth nondimensional natural frequency. This small difference is believed to be due to the use of the full $3 \times 3$ integration rule in [13].

Table 1. Non-dimensional natural frequencies $\bar{\omega}$ for a SSSS $\left[0^{\circ} / \mathbf{9 0}^{\circ}\right]_{s}$ square plate.

\begin{tabular}{cccc}
\hline $2 \times 2$ & $5 \times 5$ & Ref. [13] & $\% \Delta 5 \times 5$ \\
\hline 11.9187 & 11.7364 & 11.77252 & -0.31 \\
22.2978 & 21.8645 & 21.83344 & 0.14 \\
28.0998 & 27.3512 & 27.37726 & -0.1 \\
34.466 & 33.2477 & 33.23205 & 0.05 \\
44.7714 & 37.9417 & 37.43603 & 1.35 \\
\hline
\end{tabular}

The sensitivity of the fundamental eigenvalue to random changes in mechanical properties is illustrated in Fig. 4. The analysis in [13] is taken up to a COV value of 0.2 for the mechanical properties, while the material density and ply orientation angles are assumed deterministic. The calculated curve of the present work under these conditions is almost identical to that reported in [13]. Two other curves are presented on the figure; one with the density taken as a random variable, and the other with both the density and ply angles as random variables. It is clear from the figure that the randomness in material 
density can not be ignored, whereas randomness in ply angles has almost no effect on the randomness of the fundamental natural frequency.

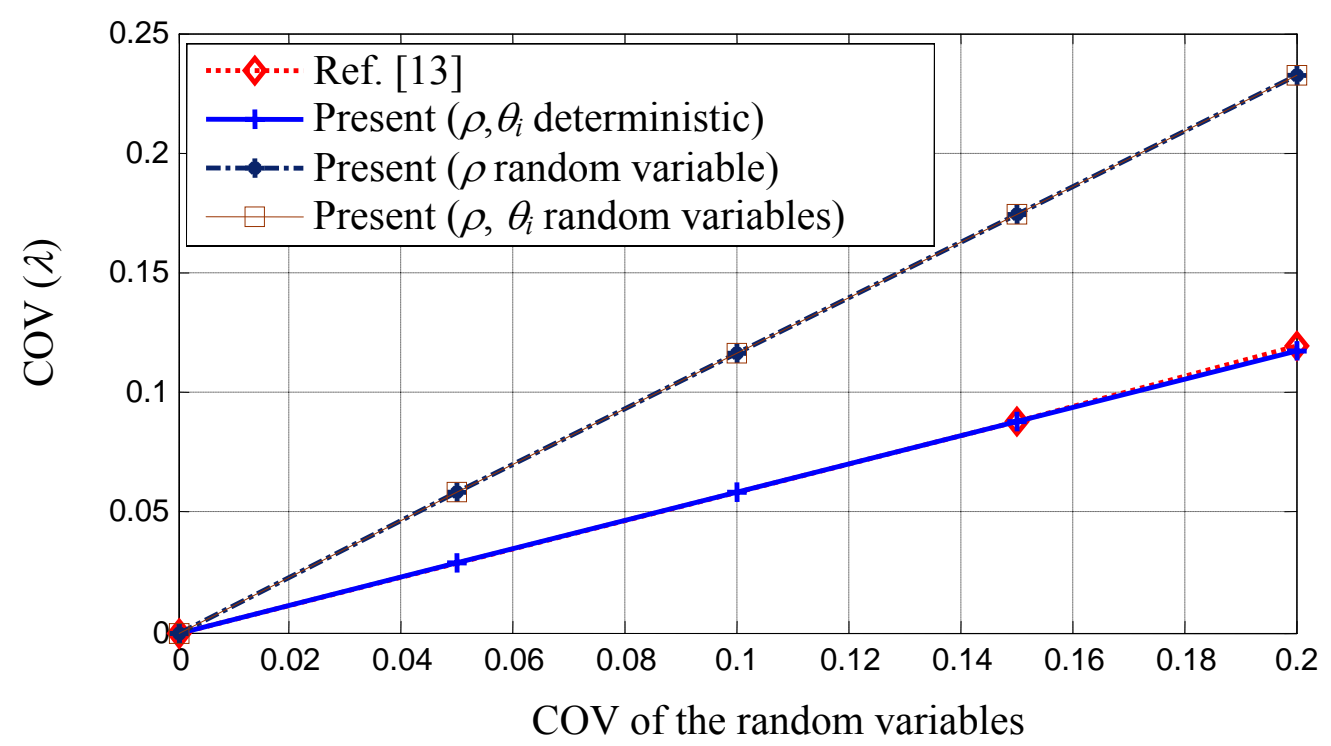

Fig. 4.Variation of $\operatorname{COV}(\lambda)$ with simultaneous changes of the random variables for a SSSS $\left[0^{\circ} / 90^{\circ}\right]_{s}$ square laminate.

The relative importance of randomness in each material property is illustrated in Fig. 5. Here $\operatorname{COV}(\lambda)$ is plotted against the $\mathrm{COV}$ of each random variable assuming all remaining variables to be deterministic at their mean values. It is clear from the figure that the natural frequency is sensitive to the following material properties, in decreasing order of importance: $\rho, E_{11}, G_{23}, G_{13}, E_{22}, G_{12}$ and $v_{12}$.

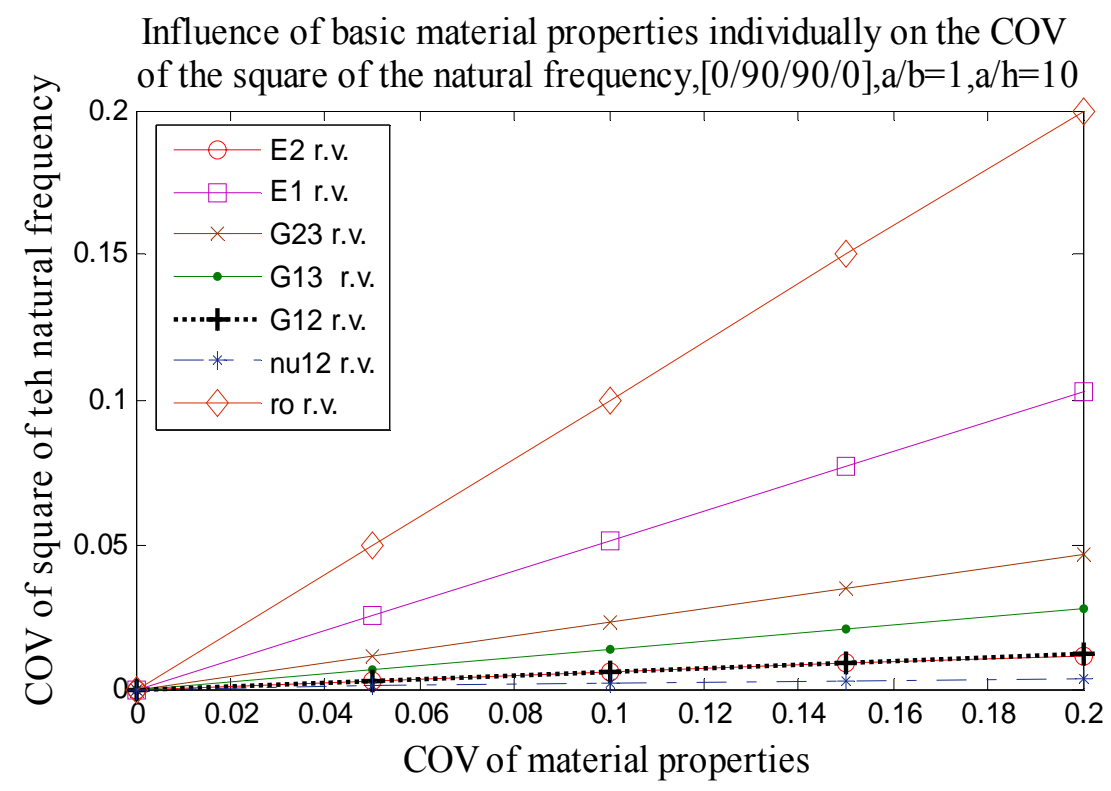

Fig. 5. Variation of $\operatorname{COV}(\lambda)$ with individual changes of the random variables for a SSSS $\left[0^{\circ} / 90^{\circ}\right]_{s}$ square laminate. 
To validate the calculated results of the present work in cases involving asymmetric laminates and different boundary conditions, a square $\left[0^{\circ} / 45^{\circ} /-45^{\circ} / 90^{\circ}\right]$ laminate made of the same material is considered. The laminate was studied in [15] for two values $a / h=5$ and $a / h=10$, and for two sets of boundary condition, SSSS and CFCF. Table 2 shows a very good agreement between the calculated nondimensional natural frequency of the present FEM scheme and the published results. Fig. 6 shows a comparison of the calculated $\operatorname{COV}(\lambda)$ for the SSSS case, based on deterministic density and ply angles, to that reported in [15]. The excellent agreement of Figs. 5 and 6 proves the validity of the present work for asymmetric, as well as, for symmetric laminates. The upper curve in Fig. 6 suggests that, unlike the symmetric case, the error in calculating $\operatorname{COV}(\lambda)$ by assuming ply angles to be deterministic in asymmetric laminates is considerably large.

Table 2. Non-dimensional fundamental frequency $\bar{\omega}$ for SSSS and CFCF $[0 \% 45 \%$ $45^{\circ} / 90{ }^{\circ}$ ] square laminates.

\begin{tabular}{ccccccc}
\hline$a / h$ & \multicolumn{3}{c}{ SSSS } & \multicolumn{3}{c}{ CFCF } \\
& Present & Ref[15] & $\Delta \%$ & Present & Ref[15] & $\Delta \%$ \\
\hline 5 & 7.3021 & 7.17 & 1.8 & 7.5926 & 7.38 & 2.9 \\
10 & 8.92 & 8.89 & 0.34 & 11.3582 & 11.1 & 2.3 \\
\hline
\end{tabular}

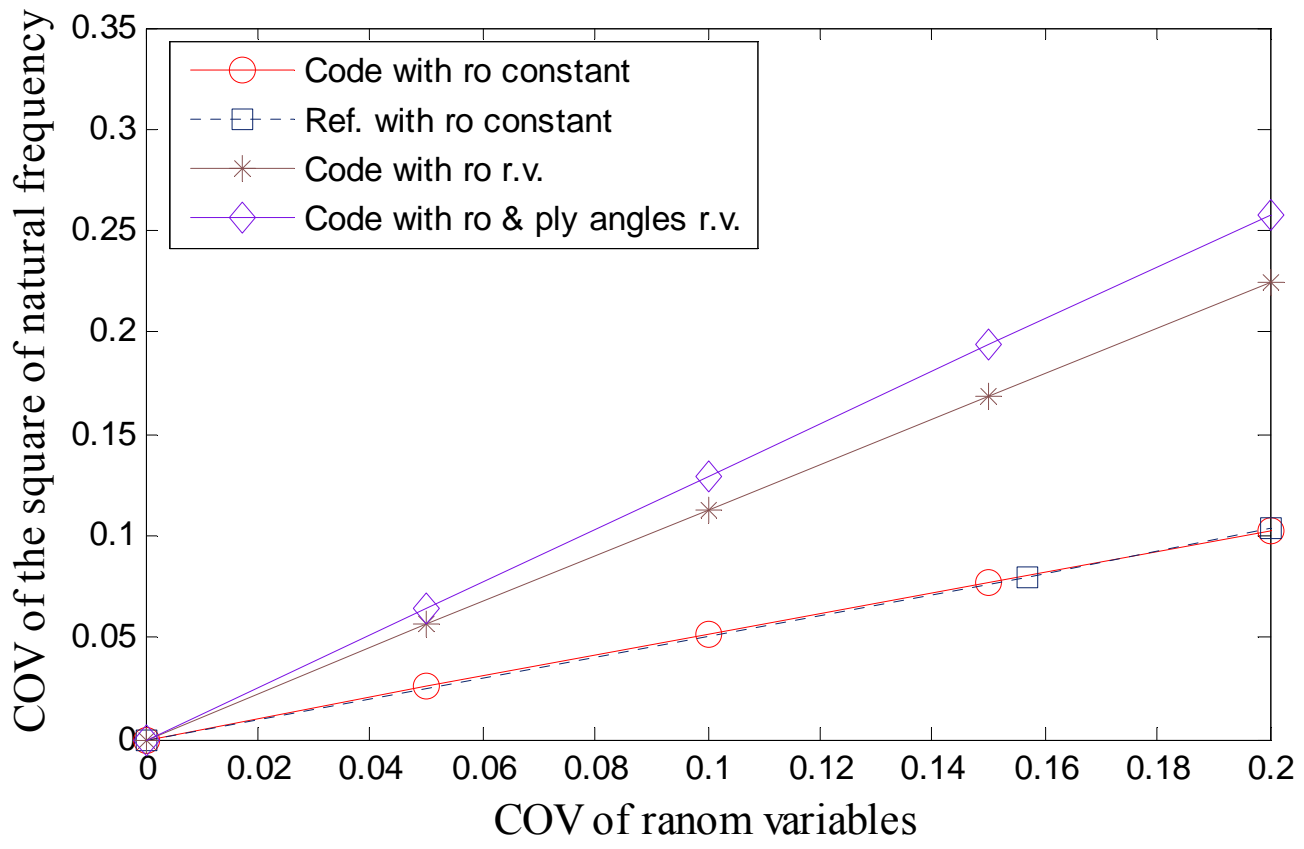

Fig. 6.Variation of $\operatorname{COV}(\lambda)$ with simultaneous changes of the random variables for a SSSS $\left[0^{\circ} / 45^{\circ} /-45^{\circ} / 90\right.$ ] square laminate. 


\section{Full Stochastic Analysis of a square asymmetric Laminate:}

Full stochastic analysis of laminates involves determining the MPP and probability of failure. To the best of our knowledge, there are no published results for this detailed stochastic finite element analysis of the free vibration of laminated composites. Most publications deal with static problems only, $[8,10,11]$, or calculate only second order statistics (COV), $[12,15]$. Accordingly, results of the present work are compared to those of the well-established Monte Carlo simulation technique. To reduce number of sufficient sample simulation, a technique that utilizes importance sampling is used. The procedure of applying this technique is detailed in [16].

The illustration is of a $\left[0^{\circ} / 45^{\circ} /-45^{\circ} / 90^{\circ}\right]$ square laminate with $a / h=10$. The uncorrelated random material properties and ply angles have the normal distributions shown in Table 3. The mean and SD of stiffnesses are in GPa, and those of the density are in $\mathrm{kg} / \mathrm{m}^{3}$. It should be noted that the developed MATLAB code facilitates the choice of other types of distributions, thus providing more flexibility in quantifying the randomness of the basic variables.

Table 3. Statistical distribution of the basic random variables.

\begin{tabular}{ccccccccc}
\hline Property & $E_{11}$ & $E_{22}$ & $G_{12}$ & $G_{23}$ & $G_{13}$ & $v_{12}$ & $\rho$ & $\Delta \theta$ \\
\hline Mean & 16.48 & 1.4 & 0.87 & 0.45 & 0.87 & 0.334 & 1,000 & $0.0^{\circ}$ \\
SD & 0.61 & 0.05 & 0.052 & 0.014 & 0.052 & 0.01 & 36 & $1.8^{\mathrm{o}}$ \\
\hline
\end{tabular}

Since the probability of failure using Monte Carlo simulation technique depends on the number of simulations, then $p_{f}$ can be taken as a dependent random variable, for which one can calculates a mean, a standard deviation and a skewness coefficient. Table 4 shows the variation of $p_{f}$ with the number of simulations for three values of the frequency ratio, $\omega_{r} / \omega_{p}=0.97,0.93,0.87$, respectively. It should be noted that for relatively low frequency ratios, the last two rows of Table $4, p_{f}$ is so small that convergence is not clearly visible due to round-off error. This problem does not exist in the present work because convergence is based on the value of $\beta$, which for all practical purposes, a very large number compared to any round-off error. The values of the mean, $\mathrm{SD}$, and skewness coefficient for the three $p_{f}$ distributions are listed in Table 5. The small values of SD suggest that the value of $p_{f}$ does not change much around the mean. Negative skewness coefficients mean that dispersion is more below the mean than above it. Therefore, taking the mean of Monte Carlo calculated $p_{f}$ as a reference for comparison is justified and reasonable.

Table 4. Variation of $p_{f}$ of Monte Carlo for SSSS $\left[0^{\circ} / 45^{\circ} /-45^{\circ} / 90^{\circ}\right]$ square laminate with $a / h=10$ for three values of the frequency ratio.

\begin{tabular}{cccccccccccc}
\hline \multicolumn{2}{c}{ No. of Simulations } & 50 & 100 & 150 & 200 & 250 & 300 & 350 & 400 & 450 & 500 \\
\hline \multirow{2}{*}{$p_{f}$} & $\left(\times 10^{2}\right)$ & 8.88 & 7.34 & 6.72 & 7.85 & 7.74 & 7.43 & 7.42 & 7.85 & 7.85 & 7.59 \\
& $\left(\times 10^{4}\right)$ & 5.10 & 3.87 & 4.52 & 4.85 & 5.09 & 4.75 & 4.88 & 5.11 & 5.34 & 5.23 \\
& $\left(\times 10^{10}\right)$ & 1.99 & 1.27 & 1.19 & 1.48 & 1.60 & 1.44 & 1.57 & 1.68 & 1.823 & 1.826 \\
\hline
\end{tabular}


Table 5. Statistical Parameters of the $p_{f}$ distributions of Table 4.

\begin{tabular}{cccc}
\hline$\omega_{r} / \omega_{p}$ & Mean & SD & $\begin{array}{c}\text { Skewness } \\
\text { Coefficient }\end{array}$ \\
\hline 0.97 & 0.0765 & $1.964 \mathrm{E}-3$ & $-9.614 \mathrm{E}-2$ \\
0.93 & $4.86 \mathrm{E}-4$ & $4.21 \mathrm{E}-5$ & -1.06 \\
0.87 & $1.58 \mathrm{E}-10$ & $2.35 \mathrm{E}-11$ & -276 \\
\hline
\end{tabular}

Table 6 shows a comparison of the safety index and the probability of failure calculated using FORM and SORM optimization methods to the mean values of Monte Carlo simulation results at the three values of the frequency ratio. For both optimization methods, solution is performed once by updating the eigenvectors at each iteration, ver(1), and another time with the eigenvectors calculated only at the mean values of the random variables and updated once at the MPP, ver(2). The last row of the table provides a comparison of the CPU time of both versions of SORM and Monte Carlo for the case of $\omega_{r} / \omega_{p}=0.97$.

Table 6. Comparison of the safety index and probability of failure of SSSS $\left[0^{\circ} / 45^{\circ} /-\right.$ $45^{\circ} / 90^{\circ}$ ] square laminate for three values of the frequency ratio.

\begin{tabular}{|c|c|c|c|c|c|}
\hline & \multicolumn{2}{|c|}{ FORM } & \multicolumn{2}{|c|}{ SORM } & \multirow[t]{2}{*}{ MonteCarlo } \\
\hline & $\operatorname{ver}(1)$ & $\operatorname{ver}(2)$ & $\operatorname{ver}(1)$ & $\operatorname{ver}(2)$ & \\
\hline \multirow{3}{*}{$\beta$} & 1.3267 & 1.3268 & 1.4103 & 1.4102 & 1.4293 \\
\hline & 3.1918 & 3.1920 & 3.2952 & 3.2951 & 3.2985 \\
\hline & 6.1858 & 6.1859 & 6.2937 & 6.2945 & 6.2905 \\
\hline \multirow{3}{*}{$P_{f}$} & 0.0923 & 0.0923 & 0.0792 & 0.0792 & 0.0765 \\
\hline & 7.07E-4 & $7.06 \mathrm{E}-4$ & $4.92 \mathrm{E}-4$ & $4.91 \mathrm{E}-4$ & $4.86 \mathrm{E}-4$ \\
\hline & $3.09 \mathrm{E}-10$ & $3.09 \mathrm{E}-10$ & $1.55 \mathrm{E}-10$ & $1.54 \mathrm{E}-10$ & $1.58 \mathrm{E}-10$ \\
\hline CPU time (s) & & & 4,674 & 2,484 & 59,616 \\
\hline
\end{tabular}

Taking the probability of failure obtained Using Monte Carlo simulation as a reference, it is concluded that using FORM overestimates $p_{f}$ by an unacceptably high percentage that increases as the frequency ratio decreases. Using SORM, on the other hand, overestimates $p_{f}$ by a maximum value of only $3.5 \%$. This indicates that the problem is highly nonlinear, and ignoring its nonlinearity in FORM introduces large errors. Table 6 also shows that results of ver(1) and ver(2) for any given optimization method are virtually the same. This confirms the validity of the assumption that the values of the eigenvectors do not change much around the mean value. Finally, the table shows the considerable save in CPU time when using the present SORM analysis. Although the importance sampling method converges to $p_{f}$ using relatively small number of simulations, its CPU time is about 13 times that of ver(1), and 24 times that of ver(2) of the almost equally accurate SORM method.

Results of the MPP for this plate are presented in Table 7. Results corresponding to $\omega_{r} / \omega_{p}=0.97$ are obtained using ver(1) and ver(2) solutions. Again, it is clear that the simplifying assumption of constant eigenvectors close to MPP is justified, even for 
relatively low reliability problems. Therefore only ver(1) results are listed for the remaining frequency ratios.

Table 7. MPP for SSSS $\left[0^{\circ} / 45^{\circ} /-45^{\circ} / 90^{\circ}\right]$ square laminate with $a / h=10$ for three values of the frequency ratio.

\begin{tabular}{cccccccccccc}
\hline$\omega_{r} / \omega_{p}$ & $E_{11}$ & $E_{22}$ & $G_{12}$ & $G_{23}$ & $G_{13}$ & $v_{12}$ & $\rho$ & $\theta_{1}$ & $\theta_{2}$ & $\theta_{3}$ & $\theta_{4}$ \\
\hline 0.97 & 16.307 & 1.3825 & 0.8398 & 0.4496 & 0.8644 & 0.3337 & 1036.4 & 0.263 & 44.519 & -45.481 & 90.270 \\
& 16.305 & 1.3826 & 0.8399 & 0.4496 & 0.8641 & 0.3337 & 1036.6 & 0.258 & 44.525 & -45.480 & 90.258 \\
0.93 & 16.057 & 1.3549 & 0.7927 & 0.4490 & 0.8567 & 0.3332 & 1085.1 & 0.596 & 43.837 & -46.163 & 90.596 \\
0.87 & 15.647 & 1.3031 & 0.7051 & 0.4482 & 0.8453 & 0.3326 & 1156.1 & 1.003 & 42.777 & -47.223 & 91.003 \\
\hline
\end{tabular}

\section{Neglecting randomness in the stacking sequence is widely used. To study the effects of this assumption (Combine tables 4.18, 4.18 and table 7 and table 4.22)}

\section{Discussion and Conclusions}

In the present study, a procedure and a MATLAB code for performing reliability analysis of the free vibration of laminated composite plates has been developed. The suggested stochastic analysis is performed using both the First Order Reliability Method (FORM-Method 2) and the Second Order Reliability Method (SORM), while the system response used FEM formulation with a nine-node isoparametric Lagrangian element. The code used MATLAB's symbolic capabilities to obtain the derivatives of the performance function symbolically, thus greatly reducing calculation time. Validity of the obtained results was established by comparisons with available published work, and algorithm effectiveness was established by comparisons with results of Monte Carlo simulation with importance sampling.

Upon investigating the sensitivity of the used performance function to random changes in material properties, it was concluded that the randomness in material density can not be neglected in any accurate analysis of the scatter in the natural frequencies of rectangular laminates. The randomness in ply angles, however, can be neglected for the cases of symmetric rectangular laminates.

Results showed that calculating the probability of failure using SORM is an excellent rapid tool in the stochastic analysis of free vibration of composite plates, when compared to the slower Monte Carlo simulation techniques, which require solving a large FEM problem so many times. Assuming the eigenvectors to be constant at the mean value of the random variables during the computation of the derivatives of the eigenvalues proved to be a reasonable simplifying assumption, which gives excellent results with much less computational time. One limitation on the probability of failure computed using FORM was emphasized; namely its large error in non-linear problems.

\section{References}


1. Nigam, N. C., and Naravanan, S., Applications of Random Vibrations, Narosa, New Delhi, 1994.

2. Ibrahim, R. A., "Structural Dynamics with Parameter Uncertainties," Applied Mechanics Reviews, Vol. 40, No. 3, pp. 309-328, 1987.

3. Manohar, C. S. and Ibrahim, R. A., "Progress in Structural Dynamics with Stochastic Parameter Variations," Applied Mechanics Reviews, Vol. 52, No. 5, pp. 177-196, 1999.

4. Oh, D. H. and Librescu, L., "Free Vibration and Reliability of Composite Cantilevers Featuring Uncertain Properties," Reliability Engineering and System Safety, Vol. 56, pp. 265-272, 1997.

5. Salim, S., Yadav, D., Iyengar, N. G. R., "Deflection of Composite Plates with Random Material Characteristics," Proceedings of Symposium on Recent Advances in Aerospace Science and Engineering Conference, Vol. 1, Bangalore, New Delhi, pp. 236-239, 1992.

6. Salim, S., Yadav, D., Iyengar, N. G. R., "Analysis of Composite Plates with Random Material Characteristics," Mechanics Research Communications, Vol. 20, No. 5, pp. 405-414, 1993.

7. Salim, S., "Analysis of Composite Plates with Randomness in Material Properties," PhD thesis, Dept. of Aerospace Engineering, IIT Kanpur, 1995.

8. Falsone, G. and Impollonia, N., "A new Approach for the Stochastic Analysis of Finite Element Modelled Structures with Uncertain Parameters," Comput. Methods Appl. Mech. Engrg., Vol. 191, pp. 5067-5085, 2002.

9. Abumeri, G. H. and Chamis, C. C.," Non-deterministic Design of Composite Structures with Uncertainties," $43^{\text {rd }}$ AIAA/ASME/ASCE/AHS/ASC Structures, Structural Dynamics\& Materials Conference, Denver, Colorado, April 2002.

10. Abumeri, G. H. and Chamis, C. C.," Non-deterministic Optimization of Composite Structures Reliability," $45^{\text {th }}$ AIAA/ASME/ASCE/AHS/ASC Structures, Structural Dynamics\& Materials Conference, Palm Springs, California, April 2004.

11. Elseifi, M.A., "Reliability formulation of thick composite plates subjected to first plyfailure". AIAA-2006-2152, 2006.

12. Shankara, C. A. and Iyengar, N. G. R., "A $C^{0}$ Element for the Free Vibration Analysis of Laminated Composite Plates," J. Sound Vib. Vol. 191, pp. 721-738, 1996.

13. Singh, B. N., Yadav, D., and Iyengar, N. G. R., "Natural Frequencies of Composite Plates with Random Material Properties Using Higher Order Shear Deformation Theory," Int. J. Mech. Sci. Vol. 43, pp. 2193-2214, 2001.

14. Hasselman, T.K., and Hart, G.C., "Modal analysis of random structural systems", $J$. of the Eng. Mechanics Division, ASCE, Vol.98, pp.561-573, 1972.

15. Singh, B. N., Yadav, D., and Iyengar, N. G. R., "A C ${ }^{0}$ Element for Free Vibration of Composite Plates with Uncertain Material Properties," Adv. Composite Mater. Vol. 11, No. 4, pp. 331-350, 2003.

16. Melchers, R.E., Structural reliability Analysis and Prediction, New York, John Wiley \& Sons, 1999.

17. Haldar, A. and Mahadevan, S., Probability, Reliability and Statistical Methods in Engineering Design, New York, John Wiley \& Sons, 2000.

18. Breitung, K. "Asymptotic Approximations for Multinormal Integrals," Journal of Engineering Mechanics, ASCE, 110(3):357-366, 1984. 
